\title{
Malófagos de galinhas-d'angola (Numida meleagris, L. 1758) em criações extensivas no estado do Rio de Janeiro
}

\section{Chewing lice of free reared guinea-fowls (Numida meleagris, L. 1758) in the state of Rio de Janeiro}

\author{
Jorge Aguiar Amaral, ${ }^{*}$ Dalton Garcia de Mattos Jr., ${ }^{* *}$ Rodrigo Caldas Menezes, ${ }^{* \star *}$ Michel Paiva Valim(***
}

\begin{abstract}
Resumo
Um estudo sobre os principais malófagos de 35 galinhas-d'angola provenientes de cinco municípios do estado do Rio de Janeiro (Barra Mansa, Maricá, Itaboraí, Cachoeira de Macacu e Cambuci) foi realizado a partir da pesquisa de penas, coleta de piolhos, contagem de espécimens, acondicionamento em álcool 70\%GL. Das 35 aves estudadas, 100\% apresentaram-se parasitadas. Oito espécies foram e suas respectivas prevalências foram: Menopon galinae (100\%); Menacanthus stramineus (2,8\%); Menacanthus pallidulus (2,8\%); Colpocephalum turbinatum (2,8\%); Lipeurus caponis (31,4\%); Lipeurus tropicalis $(2,8 \%)$; Goniodes gigas $(25,7 \%)$ e Goniocotes gallinae $(40 \%)$. O poliparasitismo foi observado, sendo a associação, Menopon gallinae $x$ Goniocotes gallinae, a mais prevalente. $O$ contato das galinhas-d'angola com diferentes espécies de aves domésticas pode ter propiciado o parasitismo de malófagos comuns às outras espécies de aves. Pela primeira vez no Brasil são registrados em galinhas-d'angola espécies de piolhos Menacanthus stramineus, Menacanthus pallidulus, Colpocephalum turbinatum, Lipeurus caponis, Lipeurus tropicalis e Goniodes gigas. As espécies Menacanthus pallidulus, Colpocephalum turbinatum e Lipeurus tropicalis foram registradas pela primeira vez neste hospedeiro, na literatura mundial.
\end{abstract}

Palavras-chave: galinha-d'angola, malófagos, criação extensiva.

\begin{abstract}
A total of 35 guinea-fowls, Numida meleagris, were examined for lice at five municipalities in the Rio de Janeiro State, Brazil, (Barra Mansa, Maricá, Itaboraí, Cachoeiras de Macacú e Cambuci). All birds were infested by lice. Eigth chewing lice species were encountered and their respective prevalence were found: Menopon galinae (100\%); Menacanthus stramineus $(2,8 \%)$; Menacanthus pallidulus (2,8\%); Colpocephalum turbinatum (2,8\%) Lipeurus caponis (31,4\%); Lipeurus tropicalis $(2,8 \%)$; Goniodes gigas $(25,7 \%)$ and Goniocotes gallinae $(40 \%)$. The polyparasitism was observed being the association between Menopon gallinae and Goniocotes gallinae, the most prevalent one. The contact among guinea-fowls and other domestic birds might have been responsible for the parasitism with lice common to other bird species. For the first time it was registered in Brazil the occurrence of the following chewing lice in guinea-fowls: Menacanthus stramineus, Menacanthus pallidulus, Colpocephalum turbinatum, Lipeurus caponis, Lipeurus tropicalis and Goniodes gigas. The chewing lice species: Menacanthus pallidulus, Colpocephalum turbinatum and Lipeurus tropicalis were registered for the first time in guinea-fowls.
\end{abstract}

Keywords: guinea-fowl, chewing lice, free reared.

\section{Introdução}

A galinha-d'angola (Numida meleagris Linnaeus, 1758) é uma ave da ordem Galliformes e da Família Phasianidae (Storer et al., 1984), originária da África. Nas criações populares, em sistema extensivo, essas aves estão sujeitas ao ataque de diversas espécies de parasitos, sendo os helmintos e os malófagos, os mais freqüentes. (Hafez e Madbooly, 1966; Aguirre et al., 1991).
Os malófagos das aves, conhecidos como piolhos mastigadores, pertencem à ordem Phthyraptera, que se divide em quatro subordens, a saber, Anoplura, Amblycera, Ischnocera e Rhyncophthirina (Clay,1970; Wall e Shearer, 1997), sendo parasitos de aves domésticas os componentes da família Menoponidae (subordem Amblycera), que engloba como principais gêneros: Menacanthus, Menopon, Holomenopon e Colpocephalum e os da família Philopteridae (subordem Ischnocera) representada principalmente pelos gêneros:

\footnotetext{
* Mestrando do Programa de Pós-Graduação em Medicina Veterinária - Patologia Veterinária - Ornitopatologia - Faculdade de Veterinária UFF - Niterói - RJ

** Professor da disciplina de Doenças Parasitárias, Departamento de Saúde Coletiva Veterinária e Saúde Pública - UFF - Niterói - RJ

*** Médico-veterinário, Centro de Criação de Animais de Laboratório - CECAL, do Instituto Oswaldo Cruz - FIOCRUZ - Rio de Janeiro - RJ

**** Médico-veterinário, Laboratório de Ixodides, Departamento de Entomologia do Instituto Oswaldo Cruz - FIOCRUZ - RJ
} 
Goniocotes, Goniodes, Clucotogaster, Lipeurus, Oxylipeurus, Columbícola, Chelopistes e Anaticola.

Sendo importantes parasitos de aves, os malófagos atingem diversas espécies de aves industriais. Pelo menos 10 espécies ocorrem em galinhas, 3 em perus, 2 em patos e 1 em codorna doméstica, sendo ainda pouco conhecidas no Brasil. (Figueiredo et al., 1993; Pinto et al., 2001).

Existem vários trabalhos sobre piolhos de galinhas domésticas em diversos países com destaque para os de Clay (1949 e 1970) e Emerson (1956). No Brasil destacam-se Vaz (1935), Oliveira e Ribeiro (1990), Figueiredo (1993), Oliveira et al. (1999), Pinto (2001) e Silva (2002).

A grande maioria dos trabalhos sobre malófagos de galinhasd'angola foram realizados em aves provenientes do continente africano, com destaque para os trabalhos de Tendeiro (1988, 1989 e 1994); Louw et al. (1993) e Price et al. (2003).

O único trabalho de que se tem conhecimento, sobre malófagos em galinhas-d'angola no Brasil, é de Ribeiro et al. (2003), que em nota prévia e sem mencionar o número de aves pesquisadas, registraram no Rio Grande do Sul, a ocorrência de Goniocotes gallinae, Menopon gallinae e Lipeurus sp, sem especificar qual espécie encontrada deste último gênero.

O presente trabalho teve como objetivo realizar um levantamento da fauna de malófagos de galinhas-d'angola criadas extensivamente, em cinco municípios do estado do Rio de Janeiro, chamando a atenção para a identificação das espécies mais prevalentes, uma vez que os piolhos podem ser considerados importantes agentes causadores de prejuízos às criações e à manutenção de lotes saudáveis adequados ao consumo.

\section{Material e métodos}

Foram examinadas 35 galinhas-d'angola (Numida meleagris) adultas e jovens, sendo 22 machos e 13 fêmeas, criadas extensivamente, adquiridas em cinco municípios do estado do Rio de Janeiro, de regiões fisiogeográficas diferentes, perfazendo um total de 7 aves por município.

Após a escolha das aves, elas foram transportadas em gaiolas diretamente para o Laboratório de Doenças Parasitárias, do Departamento de Saúde Coletiva Veterinária e Saúde Pública, da Faculdade de Veterinária da Universidade Federal Fluminense, onde se procedeu a eutanásia, de acordo com os métodos preconizados dentro da ética (Sozed, 1996), pois estes mesmos animais foram utilizados para outras pesquisas.

Procedeu-se a retirada manual das penas, que com o intuito de se obter o maior número possível de espécimens de malófagos, foram escolhidas de diferentes regiões do corpo, com preferência para o pescoço, peito, em torno da cloaca, face ventral das asas e dorso, como preconizado por Silva (2002).

As penas foram depositadas em frascos de boca larga com etanol a $70 \% \mathrm{GL}$. Todo o material foi identificado e etiquetado, a fim de prepará-los para a posterior identificação.

Posteriormente procedeu-se a clarificação dos espécimens em lactofenol e montagem entre lâmina e lamínula com bálsamo do Canadá. A identificação dos espécimens foi feita de acordo com as chaves de Emerson (1956) e Price e Graham (1996).

Os exemplares foram depositados na coleção do Laboratório de Doenças Parasitárias Animais Domésticos, do Departamento de Saúde Coletiva Veterinária e Saúde Pública, da Universidade Federal Fluminense - Niterói, RJ.

\section{Resultados e discussão}

Das 35 aves examinadas, 100\% apresentaram-se parasitadas por uma ou mais espécies de malófagos, totalizando 445 espécimens, distribuídos entre oito espécies na população de aves estudada. Esses números se assemelham aos encontrados por Louw et al. (1993) na África do Sul, apesar de que somente a espécie Goniodes gigas foi comum aos dois trabalhos, pois as espécies africanas constantes das chaves de identificação organizadas por Tendeiro (1989) não foram registradas no presente trabalho.

As espécies identificadas no presente levantamento e pela primeira vez registradas parasitando galinhas-d'angola no estado do Rio de Janeiro foram as seguintes:

I - Da família Menoponidae: Menopon gallinae (Linnaeus,1758) e Menacanthus pallidulus (Neumann,1912), que possuem maior especificidade para galinha doméstica (Gallus gallus), Menacanathus stramineus (Nistzch, 1818) que possui maior especificidade para perus (Meleagris gallopavo), e Colpocephalum turbinatum (Denny, 1842), que possui maior especificidade para pombos (Columba livia).

II - Da família Philopteridae: Lipeurus caponis (Linnaeus, 1758), Lipeurus tropicallis Peters 1931, Goniodes gigas (Taschenberg,1879) e Goniocotes gallinae (De Geer,1778), todos com maior especificidade para galinha doméstica.

Na Figura 1, observa-se que os municípios de Barra Mansa e Cambuci apresentaram o registro de seis diferentes espécies de malófagos parasitando as aves, totalizando cada um, 75\% das espécies identificadas no presente levantamento. Isto pode ser devido às condições ambientais em que as aves viviam, pois as galinhas-d'angola possuem hábitos gregários, vivem muito próximas, e grandes quantidades de indivíduos são encontrados na população, facilitando assim a infestação de piolhos através do contato direto, conforme observaram Price et al., 2003.

Da mesma forma, a ocorrência de quatro mesmas espécies igualmente em Maricá e Cambuci, regiões fisiogeográficas bem diferentes, mostra a boa capacidade de adaptação deste parasito.

No que se refere à ocorrência de aves sendo parasitadas por mais de uma espécie de malófagos, 22 (62,8\%) das aves estudadas apresentaram poliparasitismo e os municípios de Maricá $(71,4 \%)$ e Cambuci $(71,4 \%)$ foram os que apresentaram maior ocorrência. Associações entre $M$. gallinae e G. gallinae foram as mais freqüentes. O poliparasitismo foi citado como sendo tradicional em galinhas-d'angola por Idi et al. (2001), na Nigéria e já havia sido registrado por Keler (1952) em Moçambique, Louw et al. (1993) na África do Sul, Tendeiro (1994) em Angola, sendo que o presente estudo faz esse registro pela primeira vez no Brasil. 
Figura 1: Distribuição percentual de espécies de piolhos de galinhas-d'angola em função de sua ocorrência e percentual de poliparasitismo nos diferentes municípios do estado do Rio de Janeiro, 2003

\begin{tabular}{c|c|c|c|c}
\hline \hline Município & $\begin{array}{c}\text { No de } \\
\text { aves }\end{array}$ & Piolhos & $\begin{array}{c}\text { № de } \\
\text { espécies/ } \\
\text { Município }\end{array}$ & $\begin{array}{c}\text { № de aves } \\
\text { poliparasitadas/ } \\
\text { Município }\end{array}$ \\
\hline \hline Barra & 7 & $\begin{array}{c}\text { Menopon gallinae } \\
\text { Menacanthus stramineus } \\
\text { Lipeurus caponis } \\
\text { Lipeurus tropicallis } \\
\text { Goniodes gigas } \\
\text { Goniocotes gallinae }\end{array}$ & 6 (75\%) & $4(57,1 \%)$ \\
\hline Itaboraí & 7 & $\begin{array}{c}\text { Menopon gallinae } \\
\text { Lipeurus caponis } \\
\text { Goniodes gigas } \\
\text { Goniocotes gallinae }\end{array}$ & $4(50 \%)$ & $5(71,4 \%)$ \\
\hline Cachoeiras \\
de Macacu
\end{tabular}

De acordo com a Figura 2, observa-se que, das espécies identificadas no presente trabalho, M. gallinae foi a mais prevalente $(100 \%)$ e freqüente $(76,1 \%)$, o que não é normalmente registrado em galinha-d'angola pela literatura internacional. (Ayeni et al.,1983; Louw et al.,1993 e Idi et al., 2001).

A alta prevalência e freqüência de $M$. gallinae em galinhas-d'angola seguiu uma tendência semelhante ao observado em galinhas domésticas (Emerson,1956; Hafez e Madbouly, 1966; Freire, 1967/1968; Martin,1974; Torres et al., 1974; Trivedi et al., 1991; Figueiredo et al., 1993; Oliveira et al., 1999; Pinto et al., 2001 e Silva, 2002). Tal fenômeno pode estar relacionado com o sistema de criação extensivo onde as duas espécies de aves coexistem, uma vez que $M$. gallinae tem maior especificidade por galinha doméstica, segundo Price et al. ( 2003 ).

Foi observada alta prevalência para L. caponis $(31,4 \%)$, malófago comum de galinha doméstica, se comparada à encontrada por Idi et al. (2001), que observou em 190 galinhas-d'angola uma prevalência de 5,3\%, na Nigéria.

Observa-se na Figura 2 que, em relação à prevalência de infestações por diferentes espécies de malófagos em função do sexo das aves, as fêmeas se apresentaram parasitadas por um maior número de espécies. Em relação às três espécies que foram comuns às aves de ambos os sexos, os machos tiveram maior prevalência para duas dessas espécies.

Figura 2: Prevalência, Freqüência relativa, Freqüência absoluta, Amplitude de variação e Prevalência em função do sexo das aves (22 aves macho e 13 aves fêmea), de espécies de piolhos coletados em 35 de galinhas-d'angola em cinco municípios do estado do Rio de Janeiro, 2003

\begin{tabular}{|c|c|c|c|c|c|}
\hline Espécie de piolho & Prevalência & $\begin{array}{c}\text { Freqüência } \\
\text { Relativa } \\
\end{array}$ & $\begin{array}{c}\text { Freqüência } \\
\text { absoluta }\end{array}$ & $\begin{array}{c}\text { Amplitude de } \\
\text { variação }\end{array}$ & $\begin{array}{c}\text { Prevalência em função } \\
\text { do sexo das aves }\end{array}$ \\
\hline Menopon gallinae & $100 \%$ & $76,1 \%$ & 339 & $1-20$ & $\begin{array}{l}\text { M } 22(100 \%) \\
\text { F } 13(100 \%) \\
\end{array}$ \\
\hline Menacanthus stramineus & $2,8 \%$ & $0,22 \%$ & 1 & $0-1$ & $\begin{array}{l}\text { M } 0(0 \%) \\
\text { F } 1(7,6 \%)\end{array}$ \\
\hline Menacanthus pallidulus & $2,8 \%$ & $0,44 \%$ & 2 & $0-2$ & $\begin{array}{l}\text { M } 0(0 \%) \\
\text { F } 6(7,6 \%)\end{array}$ \\
\hline Lipeurus caponis & $31,4 \%$ & $9,4 \%$ & 42 & $1-12$ & $\begin{array}{l}\text { M } 5(22,7 \%) \\
F \quad 6(46,1 \%) \\
\end{array}$ \\
\hline Lipeurus tropicalis & $2,8 \%$ & $0,44 \%$ & 2 & $0-2$ & $\begin{array}{l}\text { M } 0(0 \%) \\
\text { F } 1(7,6 \%)\end{array}$ \\
\hline Goniodes gigas & $25,7 \%$ & $4,4 \%$ & 20 & $1-8$ & $\begin{array}{l}\text { M } 6(27,2 \%) \\
\text { F 3 }(23 \%)\end{array}$ \\
\hline Goniocotes gallinae & $40 \%$ & $8,5 \%$ & 38 & $1-6$ & $\begin{array}{l}\text { M } 10(45,4 \%) \\
\text { F } 4(30,7 \%)\end{array}$ \\
\hline Colpocephalum turbinatum & $2,8 \%$ & $0,22 \%$ & 1 & $0-1$ & $\begin{array}{l}\text { M } 1(4,5 \%) \\
\text { F } 0(0 \%)\end{array}$ \\
\hline
\end{tabular}


Este estudo é o primeiro no Brasil que registra a distribuição percentual de espécies de malófagos em galinhas-d'angola (M. stramineus, M. pallidulus, L. caponis, L. tropicalis, Goniodes gigas e Colpocephalum turbinatum), assim como registra, pela primeira vez na literatura mundial, essa ave como novo hospedeiro de M. pallidulus, $C$. turbinatum e L. tropicalis.

Aguirre-Uribe et al. (1991) e George et al. (1992) registraram a ocorrência da espécie C. meleagridis e M. stramineus, de maior especificidade para perus, em galinhas domésticas, sendo em relação ao $M$. stramineus, fato também registrado

\section{Referências}

AGUIRRE-URIBE, L. A.; LOZOYA-SALDANA, A.; QUIÑÓNEZ-LUNA, S.; GUERRERO, R. E. Malofagos de aves domésticas en el Sureste de Coahuila, México. Folia Entomol. Mex., v. 89, p. 93-105, 1991.

AYENY, J.S.O; DIPEOLU,O.O; OKAEME, A. N. Parasitic infections of the grey-breasted helmet guinea-fowl ( Numida meleagris galeata) in Nigeria. Veterinary Parasitology, v. 12, p. 59-63, 1983.

CLAY, T. Some problems in the evolution of a group of ectoparasites. Bull. Br. Mus. (Nat. Hist.) Evolution, v. 3: p. 279-299, 1949.

CLAY, T. The Amblycera ( Phthiraptera: Insecta). Bull. Br. Mus. (Nat.Hist.) Entomol., v. 3, n. 25, p. 75-98,1970.

EMERSON, K.C. Mallophaga (chewing lice) occurring on the domestic chicken. J. Kansas Entomol. Soc., v. 29, n. 2, p. 63-79, 1956.

FIGUEIREDO, S.M.; GUIMARÃES, J. H.; GAMA, N. M. S. Q. Biologia e ecologia de malófagos (Insecta, Phthiraptera) em aves de postura de granjas industriais. Rev. Bras. Parasitol. Vet. v. 2, n. 1, p. 45-51, 1993.

FREIRE, J. J. Fauna parasitária riograndense. Rev. Fac. Agron. Vet., v. 9, p. 111-149, 1967/1968.

GEORGE, J. B. D.; OTOBO, S.; OGUNLEYE; ADEDIMINIYI. Louse and mite infestation in domestic animals in Northern Nigeria. Trop. Anim. Proc. V. 24, p. 121-124, 1992.

HAFEZ, M. e MADBOOLY, M.H. Bird lice infesting domestic animals. Bull. Soc. Entomol. Egypt., v. 50, n. 59, p. 181-213, 1966.

IDI, A.; MAIKANO, I.; BAKO, I.; GARBA, D. and NDOMBA, N. Serological and Parasitological survey on local guinea fowl at village level in Niger. Livestock Community and Environment. Proceedings of the $10^{\text {th }}$. Conference of the Association of Institutions for Tropical Veterinary Medicine.Copenhagen. Denmark. 2001.

KÉLER.S. Von. Notes on some mallophages from mammals and gallinaceous birds in Mocambique and South Africa. Moçambique Documentário Trimestral, v. 72, p. 17-62, 1952.

LOUW, J.P;HORAK, I.G;SANTAMEYER and PRICE, R.D. Lice on helmeted guineafowls at five localities in South Africa. Onderst. J. Vet. Res., v. 60, p. 223-228, 1993.

MARTIN, M.P. Observaciones sobre algunas especies de Menoponidae ( Mallophaga: Insecta) parasitas de aves domésticas en España. Vie Milieu, v. 24, n. 1, p. 151-158, 1974.

OLIVEIRA, C. M. B.; Ribeiro, V. L. Ocorrência de Menacanthus cornutus (Mallophaga: Menoponidae) em galinhas do Rio Grande do Sul. Arq. Bras. Med. Vet. Zootec, v. 42, n. 2, p. 121-126, 1990.

OLIVEIRA, H. H.; FERREIRA, I.; SERRA-FREIRE, N. M. Fauna de Mallophaga (Insecta: Phthiraptera) de ectoparasitos em Gallus gallus L. e Columba livia L. amostrados no Rio de Janeiro-Brasil. Entomol. Vect. , v. 6, n. 5, p. 509-515, 1999. por diversos outros autores. Da mesma forma, investigadores como Tendeiro (1988, 1989 e 1994) e Price et al. (2003), apresentam uma rica contribuição ao estudo dos malófagos, registrando que a ectoparasitofauna de galinhas-d'angola pode ser encontrada em aves domésticas e selvagens. Dessa forma, justifica-se a galinha-d'angola como novo hospedeiro de $M$. pallidulus e $L$. tropicalis, malófagos comuns de galinhas domésticas e $C$. turbinatum, malófago comum de pombos, sendo este último registrado por Oliveira et al. (1999), também parasitando galinhas domésticas.

PINTO, C; POSSATI, M; VILAÇA, A. GUERIM; L. SÁ-FREIRE, L.; SERRAFREIRE, N. M. Ocorrência de malófagos em galinhas caipiras e sua relação com o padrão de coloração da plumagem. Entomol. Vect., v. 8, n. 3, p. 295-301, 2001.

PRICE, M. A.; GRAHAM, O. H. Chewing and Sucking Lice as Parasites of Mammals and Birds. U.S. Department of Agriculture, Technical Bulletin n. 1849, 1996. 309 p.

PRICE, R. D.; Hellenthal, R. A.; Palma, R. L.; Johnson, K. P.; Clayton, D. $\mathrm{H}$. The chewing lice: world checklist and biological overview. Illions Natural History Survey Special Publication, v. 24. 2003. 501 p.

RIBEIRO, P. B; COSTA, P. R. P; KRUGER, R. F.;BICHO, C. L. Ocorrência de Goniocotes gallinae; Lipeurus spp e Menopon gallinae em Numida meleagris (Linnaeus, 1758) em Arroio Grande, RS, Brasil. Arq. Inst. Biol., São Paulo, v. 70, n. 2, p. 229, abr./jun. 2003.

SILVA, S. B. Dinâmica da fauna ectoparasitária Ischnocera, Amblycera e Acari) em galinhas domésticas (Gallus gallus) de criações rústicas no município de Patos - Paraíba: estudos preliminares. 2002. 68 f. Dissertação (Mestrado em Ciências Veterinárias)-Faculdade de Veterinária, Universidade Federal Rural do Rio de Janeiro, Seropédica, RJ.

STORER, T. I; USINGER, R. L;STEBBINS, R. C; NYBAKKEN, J. W. Zoologia Geral. São Paulo: Editora Nacional, 1984.816 p.

SOZED, Sociedade Zoófila Educativa. Código de ética no uso científico de animais.1996. $12 \mathrm{p}$.

TENDEIRO, J. Études Sur Les Goniodidés (Mallophaga, Ischnocera) des Galliformes. III - Espèces Parasites des Numididés. Instituto de Investigação Científica Tropical - Estudos Ensaios e Documentos, v. 149. $173 \mathrm{p}, 1988$.

TENDEIRO, J. Études sur lês Mallophages (Insecta, Mallophaga) dês Galliformes de l'Afrique centrale. I I- Genre Goniocotes Burmeister, 1838. Garcia de Orta, Ser. Zool. Lisboa, v. 15, n. 1, p. 63-98, 1988/1989. TENDEIRO, J.;MENDES, L. F.; AGUIAR, O. B. Études sur les Mallophages (Insecta, Mallophaga) des Galliformes de L'Afrique Centrale. III. Nouvelles Données sur lês Amblycera et description de 12 espèces nouvelles du genre Clayia hopkins. Instituto de Investigação Científica Tropical Estudos Ensaios e Documentos, v. 159.101 p. 1994.

TORRES, P; FRANJOLA, R.; YÁNEZ, L.; DÁZ, V.; GONZÁLEZ, E.; MONTECINOS, M. I. Estudio preliminar sobre helmintos y artrópodos del Gallus gallus domesticus en la Provincia de Valdivia, Chile. Bol. Chil. Parasitol., v. 29, p. 115-117, 1974.

TRIVED, M. C.; RAWAT, B. S.; SAXENA, A. K. The distribuition of lice (Phthiraptera) on poultry (Gallus domesticus). Int. J. Parasitol., v. 21, n. 2, p. 247-249, 1991.

VAZ. Z. Ectoparasitos de animais domésticos observados no Estado de São Paulo. Arch. Hist. Biol., v. 6, n. 2, p. 29-33, 1935.

WALL, R.; SHEARER, D. eterinary Entomology. Chapman \& Wall, p. 285-311, 1997. 